gr-qc/0212065

\title{
SCALAR FIELDS IN MULTIDIMENSIONAL GRAVITY. NO-HAIR AND OTHER NO-GO THEOREMS
}

\author{
K.A. Bronnikov ${ }^{a, b, 1}$, S.B. Fadeev ${ }^{a}$ and A.V. Michtchenko ${ }^{c, 2}$ \\ a VNIIMS, 3-1 M. Ulyanovoy St., Moscow 117313, Russia \\ ${ }^{b}$ Institute of Gravitation and Cosmology, PFUR, 6 Miklukho-Maklaya St., Moscow 117198, Russia \\ c SEPI-ESIME, IPN, Zacatenco, México, D.F., CP07738, Mexico
}

\begin{abstract}
Global properties of static, spherically symmetric configurations with scalar fields of sigma-model type with arbitrary potentials are studied in $D$ dimensions, including models where the space-time contains multiple internal factor spaces. The latter are assumed to be Einstein spaces, not necessarily Ricci-flat, and the potential $V$ includes contributions from their curvatures. The following results generalize those known in four dimensions: (A) a nohair theorem on the nonexistence, in case $V \geq 0$, of asymptotically flat black holes with varying scalar fields or moduli fields outside the event horizon; (B) nonexistence of particlelike solutions in field models with $V \geq 0$; (C) nonexistence of wormhole solutions under very general conditions; (D) a restriction on possible global causal structures (represented by Carter-Penrose diagrams). The list of structures in all models under consideration is the same as is known for vacuum with a cosmological constant in general relativity: Minkowski (or AdS), Schwarzschild, de Sitter and Schwarzschild - de Sitter, and horizons which bound a static region are always simple. The results are applicable to various Kaluza-Klein, supergravity and stringy models with multiple dilaton and moduli fields.
\end{abstract}

\section{Introduction}

Extra dimensions have become an inevitable ingredient in numerous attempts to build a unification theory including gravity [1]. The most popular dimensions are now apparently 10 (superstrings) and 11 (M-theory), although even higher dimensions are sometimes invoked [2]. The bosonic sector of such theories generically includes scalars (dilatons), and effective scalars (moduli fields) appear at dimensional reduction. A diversity of scalar fields are involved in other branches of theoretical physics and cosmology: Goldstone and Higgs fields in particle theory, inflatons and scalar dark energy models in modern cosmology, etc. It is therefore highly desirable to know the possible properties of gravitationally selfbound configurations formed by different scalar fields, and of utmost interest are conditions for the existence of black hole and solitonic, or particlelike solutions.

The simplest scalar field is massless, minimally coupled to gravity, in general, possessing a certain potential. Meanwhile, the field equations for self-gravitating scalar fields with nontrivial potentials can be explicitly integrated in very few cases, even for highly symmetric systems considered in cosmology or for static, spherically symmetric systems. Therefore, of great value are general inferences or theorems on the properties of such systems, which can be obtained without entirely solving the field equations.

For static, spherically symmetric scalar-vacuum configurations in general relativity (GR), described by the action

$$
\begin{aligned}
S=\int d^{4} x \sqrt{|g|}\left[\mathcal{R} / \kappa^{2}+L_{\mathrm{sc}}\right], & \\
L_{\mathrm{sc}} & =g^{\mu \nu} \varphi_{, \mu} \varphi_{, \nu}-2 V(\varphi),
\end{aligned}
$$

\footnotetext{
${ }^{1}$ e-mail: kb@rgs.mccme.ru

e-mail: mial@maya.esimez.ipn.mx
}

where $\mathcal{R}$ is the scalar curvature and $\kappa^{2}$ is the gravitational constant, among such theorems are:

A. The no-hair theorem [3, 4] claiming that asymptotically flat black holes cannot have nontrivial external scalar fields with nonnegative $V(\varphi)$;

B. The generalized Rosen theorem [5] asserting that a particlelike solution (i.e., an asymptotically flat solution with a regular centre) with positive mass cannot be obtained in case $V \geq 0$;

C. The nonexistence theorem for regular solutions without a centre (e.g., wormholes) [6];

D. The causal structure theorem [6], asserting that the list of possible types of global causal structures (and the corresponding Carter-Penrose diagrams) for configurations with any potentials $V(\varphi)$ and any spatial asymptotics is the same as the one for $\varphi=$ const, namely: Minkowski (or AdS), Schwarzschild, de Sitter and Schwarzschild — de Sitter.

These results will be referred to as Statements A, B, $\mathrm{C}, \mathrm{D}$, respectively.

A number of exact solutions provide examples of configurations admitted by these theorems: black hole solutions with a scalar field and $V(\varphi) \geq 0$ but with non-flat asymptotics [7], asymptotically flat black hole and particlelike solutions with negative $V(\varphi)$ [8]. All this, taken together, gives rather a clear picture of what can and what cannot be expected from static, minimally coupled scalar fields in general relativity.

There are many generalizations of the action (11) for which it is of interest to know whether or not, or under which additional requirements, Statements A-D are valid. In particular: 
1. Multidimensional configurations in GR, with $L_{\mathrm{sc}}$ given by (11) and the metric

$$
d s^{2}=\mathrm{e}^{2 \gamma} d t^{2}-\mathrm{e}^{2 \alpha} d u^{2}-\mathrm{e}^{2 \beta} d \Omega_{d_{0}}^{2}
$$

where $\alpha, \beta, \gamma$ are functions of the radial coordinate $u$ and $d \Omega_{d_{0}}^{2}$ is the linear element on the sphere $\mathbb{S}^{d_{0}}$ of unit radius.

2. More general scalar field Lagrangians in GR, e.g., $L_{\mathrm{sc}}=F(\varphi, I)$ where $I=g^{\mu \nu} \varphi_{, \mu} \varphi_{, \nu}$ and $F$ is an arbitrary function of two variables.

3. Sets of scalar fields $\vec{\varphi}=\left\{\varphi^{K}\right\}, K=\overline{1, N}$ of $\sigma$ model type, considered as coordinates in an $N$ dimensional target space $\mathbb{T}_{\varphi}$, so that

$$
L_{\mathrm{sc}}=H_{K L}(\vec{\varphi})\left(\partial \varphi^{K}, \partial \varphi^{L}\right)-2 V(\vec{\varphi})
$$

where the target space metric $H_{K L}$ (usually supposed to be positive-definite) and the potential $V$ are functions of $N$ variables $\varphi^{K}$, and we use the notation

$$
(\partial y, \partial z)=g^{\mu \nu} \partial_{\mu} y \partial_{\nu} z .
$$

4. Scalar-tensor theories (STT) of gravity, with the $D$-dimensional action

$$
S_{\mathrm{STT}}=\int d^{D} x \sqrt{g}\left[f(\phi) \mathcal{R}+h(\phi)(\partial \phi)^{2}-2 U(\phi)\right],
$$

where $(d \phi)^{2}=(\partial \phi, \partial \phi)$ and $f, h, U$ are arbitrary functions of the scalar field $\phi$.

5. High-order (curvature-nonlinear) gravity (HOG) theories, e.g., with the scalar curvature $\mathcal{R}$ in (1) replaced by a function $f(\mathcal{R})$.

6. More general multidimensional configurations, e.g., in product manifolds like

$$
\mathbb{M}^{D}=\mathbb{R}_{u} \times \mathbb{M}_{0} \times \mathbb{M}_{1} \times \mathbb{M}_{2} \times \cdots \times \mathbb{M}_{n}
$$

where $\mathbb{M}_{\text {ext }}=\mathbb{R}_{u} \times \mathbb{M}_{0} \times \mathbb{M}_{1}$ is the "external" manifold with the metric (2), $\mathbb{R}_{u} \subseteq \mathbb{R}$ is the range of the radial coordinate $u, \mathbb{M}_{1}$ is the time axis, $\mathbb{M}_{0}=\mathbb{S}^{d_{0}}$. Furthermore, $\mathbb{M}_{2}, \ldots, \mathbb{M}_{n}$ are "internal" factor spaces of arbitrary dimensions $d_{i}$, $i=2, \ldots, n$, and, according to this notation, we also have $\operatorname{dim} \mathbb{M}_{0}=d_{0}$ and $\operatorname{dim} \mathbb{M}_{1}=d_{1}=1$.

One can continue the list and/or combine its different items to obtain more generalizations.

As is clear from the previous papers [8, 9, 10, some extensions are achieved quite easily. Thus, Statements $\mathrm{C}$ and $\mathrm{D}$ are extended to items $1,2,3$ of the above list in a straightforward manner 8]. Extensions of Statements A and B generally require additional studies. Statement $\mathrm{D}$ proves to be the most universal, in particular, it holds [9, 10] in STT and HOG under very general conditions. Unlike that, Statement C is violated in STT where wormhole solutions are found quite often.

Let us discuss this point in some detail since it will be relevant in what follows.

A study of STT is effectively conducted with the aid of the well-known conformal mapping which generalizes Wagoner's [11] 4-dimensional transformation

$$
\begin{aligned}
g_{\mu \nu} & =|f(\phi)|^{-2 /(D-2)} \bar{g}_{\mu \nu}, \\
\frac{d \psi}{d \phi} & = \pm \frac{\sqrt{|l(\phi)|}}{f(\phi)}, \quad l(\phi) \stackrel{\text { def }}{=} f h+\frac{D-1}{D-2}\left(\frac{d f}{d \phi}\right)^{2},
\end{aligned}
$$

which removes the nonminimal scalar-tensor coupling expressed in the factor $f(\phi)$ before $\mathcal{R}$. The action (5), originally written in the manifold $\mathbb{M}_{J}[g]$ (the Jordan frame), will now be specified in the new manifold $\mathbb{M}_{\mathrm{E}}[\bar{g}]$ with the new metric $\bar{g}_{\mu \nu}$ (the Einstein frame) and the new scalar field $\psi$ :

$$
S_{\mathrm{E}}=\int d^{D} x \sqrt{\bar{g}}\left\{\operatorname{sign} f\left[\overline{\mathcal{R}}+(\operatorname{sign} l)(\partial \psi)^{2}\right]-2 V(\psi)\right\},
$$

where the determinant $\bar{g}$, the scalar curvature $\overline{\mathcal{R}}$ and $(\partial \psi)^{2}$ are calculated using $\bar{g}_{\mu \nu}$, and

$$
V(\psi)=|f|^{-D /(D-2)}(\psi) U(\phi) .
$$

The action (44) is similar to (11), but, in addition to arbitrary $D$, contains two sign factors. Let us suppose the usual sign of gravitational coupling, $f>0$. Then theories with $l(\phi)<0$ lead to an anomalous sign of the kinetic term of the $\psi$ field in (44) - a "ghost" scalar field as it is sometimes called. Such fields easily violate all the standard energy conditions, including the null energy condition, and consequently wormholes are quite generic (as was probably first noticed in Ref. [12] in a discussion of static, spherically symmetric solutions to the STT equations with $U=0$ ).

Wormholes, however, can even appear in normal STT, with $l(\phi)>0$, due to the so-called conformal continuations [9]. Namely, it can happen that the transformation (7) maps a singular surface in $\mathbb{M}_{E}$ to a regular surface in $\mathbb{M}_{J}$ due to a singular behaviour of the conformal factor, which compensates the singularity in $\mathbb{M}_{\mathrm{E}}$. Then $\mathbb{M}_{\mathrm{J}}$ can be continued in a regular manner through this surface (the so-called conformal continuation $[9]$ ), and the global properties of $\mathbb{M}_{\mathrm{J}}$ can be considerably richer than those of $\mathbb{M}_{\mathrm{E}}$. Ref. 10 has established necessary and sufficient conditions for the existence of conformal continuations in static, spherically symmetric systems in STT and described the properties of conformally continued solutions. It was shown, in particular, that a static, traversable wormhole is a generic result of conformal continuation.

In this paper we concentrate our attention on multidimensional theories of gravity.

In Sec. 2 we discuss the properties of self-gravitating $\sigma$-models with the Lagrangian (3) in space-times with 
the metric (2). After a brief presentation of the already known [8] Statements C and D for these systems, we give a proof of the no-hair theorem, to a large extent following the ideas of Adler and Pearson [沟. Then, we strengthen Statement B, showing that a particlelike solutions of any (even negative) mass cannot be obtained in a field model with a nonnegative potential $V(\vec{\varphi})$. This result follows from the universal identity (36), valid for all static, spherically symmetric particlelike configurations, and seems to be new even in four dimensions.

Sec. 3 is devoted to scalar field theories of $\sigma$-model type in manifolds of the form (6), often obtained in the low-energy limit of supergravities, string theories and their generalizations [1, 2]. These scalar field theories are reduced to the field model studied in Sec.2, with a target space supplemented by moduli fields (scale factors of extra dimensions), and accordingly the results of Sec. 2 are extended to these more general theories with certain natural modifications. Moreover, since the theory constructed in $\mathbb{M}^{D}$ (6) may be formulated in different conformal frames, so that the action takes a form similar to (5), we briefly discuss the conformal frame dependence of the previous results.

The Appendix contains expressions for some geometric quantities used in the previous sections and explicit formulations of the regular centre and flat asymptotic conditions for multidimensional space-times.

Throughout the paper all relevant functions are assumed to be sufficiently smooth, unless otherwise explicitly indicated.

\section{Spherically symmetric systems in $D$-dimensional GR with a $\sigma$-model source}

\subsection{Field equations}

Consider $D$-dimensional GR with the set of scalar fields (3). The Einstein equations can be written in two equivalent forms:

$$
\begin{aligned}
& \quad G_{\mu}^{\nu} \stackrel{\text { def }}{=} \mathcal{R}_{\mu}^{\nu}-\frac{1}{2} \delta_{\mu}^{\nu} \mathcal{R}=-\kappa^{2} T_{\mu}^{\nu}, \\
& \text { or } \quad \mathcal{R}_{\mu}^{\nu}=-\kappa^{2} \widetilde{T}_{\mu}^{\nu} \stackrel{\text { def }}{=}-\kappa^{2}\left(T_{\mu}^{\nu}-\frac{\delta_{\mu}^{\nu}}{D-2} T_{\alpha}^{\alpha}\right),
\end{aligned}
$$

where $T_{\mu}^{\nu}$ is the stress-energy tensor (SET), which for the fields (3) is given by

$$
T_{\mu}^{\nu}=\partial^{\nu} \vec{\varphi} \partial_{\mu} \vec{\varphi}-\frac{1}{2} \delta_{\mu}^{\nu} L_{\mathrm{sc}},
$$

or in the "tilded" form,

$$
\widetilde{T}_{\mu}^{\nu}=\partial^{\nu} \vec{\varphi} \partial_{\mu} \vec{\varphi}-\delta_{\mu}^{\nu} V(\vec{\varphi})
$$

where two arrows denote a scalar product in the target space $\mathbb{T}_{\varphi}: \vec{a} \vec{b}=H_{K L} a^{K} b^{L}$.

The static, spherically symmetric metric (2) is written with an arbitrary radial coordinate $u$. Now, it is convenient for our purposes to use the coordinate $u=\rho$ corresponding to the gauge condition $\alpha+\gamma=0$, so that the metric takes the form

$$
d s^{2}=A(\rho) d t^{2}-\frac{d u^{2}}{A(\rho)}-r^{2}(\rho) d \Omega_{d_{0}}^{2}
$$

where we have denoted $r(\rho)=\mathrm{e}^{\beta}$ and $A(\rho)=\mathrm{e}^{2 \gamma} \equiv$ $\mathrm{e}^{-2 \alpha}$. This choice is preferable for considering Killing horizons, described as zeros of the function $A(\rho)$. The reason is that in a close neighbourhood of a horizon the coordinate $\rho$ defined in this way varies (up to a positive constant factor) like manifestly well-behaved Kruskallike coordinates used for an analytic continuation of the metric [13]. Thus, using this coordinate, which may be called quasiglobal, one can "cross the horizons" preserving the formally static expression for the metric.

With this choice of the coordinate gauge, the scalar field equations and four different combinations of Eqs. (12) can be written as follows:

$$
\begin{aligned}
{\left[A r^{d_{0}} H_{K L}\left(\varphi^{L}\right)^{\prime}\right]^{\prime} } & =r^{d_{0}} \frac{\partial V}{\partial \varphi^{K}} ; \\
\left(A^{\prime} r^{d_{0}}\right)^{\prime} & =-\left(4 / d_{0}\right) r^{d_{0}} \kappa^{2} V ; \\
d_{0} r^{\prime \prime} / r & =-\kappa^{2}\left(\vec{\varphi}^{\prime}\right)^{2} ; \\
y y A\left(r^{2}\right)^{\prime \prime}-r^{2} A^{\prime \prime}+\left(d_{0}\right. & -2) r^{\prime}\left(2 A r^{\prime}-A^{\prime} r\right) \\
& =2\left(d_{0}-1\right) ; \\
d_{0}\left(d_{0}-1\right)\left(1-A{r^{\prime}}^{2}\right) & -d_{0} A^{\prime} r r^{\prime} \\
& =-A r^{2}\left(\vec{\varphi}^{\prime}\right)^{2}+2 r^{2} V .
\end{aligned}
$$

Eqs. (17), (18) and (19) are the components $\left(\begin{array}{l}t \\ t\end{array}\right),\left(\begin{array}{l}t \\ t\end{array}\right)-\left(\begin{array}{c}\rho \\ \rho\end{array}\right)$ and $\left(\begin{array}{l}t \\ t\end{array}\right)-\left(\begin{array}{l}\theta \\ \theta\end{array}\right)$, respectively, of (12), and (20) is the $\left(\begin{array}{l}\rho \\ \rho\end{array}\right)$ component of (11). We have written $(N+4)$ equations for $(N+2)$ unknowns $\varphi^{K}, A$ and $r$; but there are only two independent equations among (17)-(20), in particular, (20) is a first integral of the other equations. So this set of equations is determined.

\subsection{Global structure theorems}

One can directly extend to the present system the reasonings of Refs. [6, 8] leading to Statements C and D. Let us give, for completeness, precise formulations of the corresponding theorems.

The first theorem concerns the nonexistence of wormholes, horns and flux tubes. A wormhole is, by definition, a configuration with two asymptotics at which $r(\rho) \rightarrow \infty$, hence with $r(\rho)$ having at least one regular minimum. A flux tube is characterized by $r=$ const $>$ 0 , i.e., it is a static $\left(d_{0}+1\right)$-dimensional cylinder. A horn is a configuration that tends to a flux tube at one of its asymptotics, which happens if $r(\rho) \rightarrow$ const $>0$ at one of the ends of the range of $\rho$. Such "horned particles" with a flat asymptotic at the other end were discussed as possible remnants of black hole evaporation 14.

Theorem 1. Eqs. (10)-(20) for $D \geq 4$ and positivedefinite $H_{K L}$ do not admit (i) solutions where the func- 
tion $r(\rho)$ has a regular minimum, (ii) solutions describing a horn, and (iii) flux-tube solutions with $\varphi^{K} \neq$ const.

A proof rests on Eq. (18), implying $r^{\prime \prime} \leq 0$, which actually expresses the null energy condition valid for the SET $T_{\mu}^{\nu}$ when the matrix $H_{K L}$ is positive-definite. As a result, not only wormholes as global entities are impossible but even wormhole throats.

Another theorem concerns the possible number and order of Killing horizons, coinciding with the number and order of zeros of $A(\rho)$. A simple (first-order) or any odd-order horizon separates a static region, $A>0$ (also called an $\mathrm{R}$ region), from a nonstatic region, $A<$ 0 where (2) is a homogeneous cosmological metric of Kantowski-Sachs type (a T region). A horizon of even order separates regions with the same sign of $A(\rho)$.

The order and disposition of horizons unambiguously determine the global causal structure of space-time (up to identification of isometric surfaces, if any) 115]-18. The following theorem severely restricts such possible dispositions.

Theorem 2. Consider solutions to Eqs. (16)-(19) for $D \geq 4$. Let there be a static region $a<\rho<b \leq \infty$. Then:

(i) all horizons are simple;

(ii) no horizons exist at $\rho<a$ and at $\rho>b$.

A proof of this theorem [6, 8] employs the properties of Eq. (19), which can be rewritten in the form

$$
r^{4} B^{\prime \prime}+\left(d_{0}+2\right) r^{3} r^{\prime} B^{\prime}=-2\left(d_{0}-1\right)
$$

where $B(\rho)=A / r^{2}$. At points where $B^{\prime}=0$, we have $B^{\prime \prime}<0$, therefore $B(\rho)$ cannot have a regular minimum. So, having once become negative while moving to the left or to the right along the $\rho$ axis, $B(\rho)$ (and hence $A(\rho))$ cannot return to zero or positive values.

By Theorem 2, there can be at most two simple horizons around a static region. A second-order horizon separating two nonstatic regions can appear, but this horizon is then unique, and the model has no static region.

The possible dispositions of zeros of the function $A(\rho)$, and hence the list of possible global causal structures, are thus the same as in the case of vacuum with a cosmological constant. The latter is a solution to Eqs. (16)-(19) with $\varphi^{K}=$ const, $\kappa^{2} V=\Lambda=$ const, and the metric

$$
\begin{aligned}
& d s^{2}=A(r) d t^{2}-\frac{d r^{2}}{A(r)}-r^{2} d \Omega_{d_{0}}^{2}, \\
& A(r)=1-\frac{2 m}{\left(d_{0}-1\right) r^{d_{0}-1}}-\frac{2 \Lambda r^{2}}{d_{0}\left(d_{0}+1\right)} .
\end{aligned}
$$

This is the multidimensional Schwarzschild-de Sitter (or Tangherlini-de Sitter) solution. Its special cases correspond to the Schwarzschild $\left(d_{0}=2\right)$ and Tangherlini
19] $\left(d_{0} \geq 2\right)$ solutions when $\Lambda=0$ and the de Sitter solution in arbitrary dimension when $m=0$, called anti-de Sitter (AdS) in case $\Lambda<0$. For $\Lambda>0$, if $m$ is positive but smaller than the critical value

$$
m_{\mathrm{cr}}=\frac{d_{0}-1}{d_{0}+1}\left[\frac{d_{0}\left(d_{0}-1\right)}{2 \Lambda}\right]^{\left(d_{0}-1\right) / 2},
$$

there are two horizons, the one at smaller $r$ being interpreted as a black hole horizon and the other as a cosmological horizon. If $m=m_{\mathrm{cr}}$, the two horizons merge, and there are two homogeneous nonstatic regions separated by a double horizon. The solution with $m>m_{\mathrm{cr}}$ is purely cosmological and has no Killing horizon. In cases $m<0$ and/or $\Lambda<0$ there is at most one simple horizon. All the corresponding Carter-Penrose diagrams are well known ([20], see also [9, 10]) and will not be reproduced here.

In $(2+1)$-dimensional gravity $\left(d_{0}=1\right)$ we have a still shorter list of global structures: at most one simple horizon is possible.

Theorems 1 and 2 are independent of the form of the potential and of any assumptions about spatial asymptotics.

\subsection{No-hair theorem}

Let us now consider asymptotically flat space-times, which means, in terms of the metric (15), that, without loss of generality, $r \approx \rho$ and the function $A(\rho) \approx A(r)$ has the Tangherlini form, i.e., (23) with $\Lambda=0$ :

$$
A(r)=1-\frac{2 m}{\left(d_{0}-1\right) r^{d_{0}-1}}
$$

as $\rho \rightarrow \infty$. It then follows from the field equations that the SET components, and hence the quantities $V$ and $(\vec{\varphi})^{2}$, decay at large $\rho \approx r$ quicker than $r^{-\left(d_{0}+1\right)}$.

Let us now prove the following no-hair theorem, extending to our system the theorems known in four dimensions [3, 迎:

Theorem 3. Given Eqs. (10)-(20) for $D \geq 4$, with a positive-definite matrix $H_{K L}(\vec{\varphi})$ and $V(\vec{\varphi}) \geq 0$, the only asymptotically flat black hole solution is characterized by $V \equiv 0, \vec{\varphi}=$ const and the Tangherlini metric (22), (25) in the whole range $h<\rho<\infty$ where $\rho=h$ is the event horizon.

At the event horizon $\rho=h$ we have by definition $A=A(h)=0$, and $A>0$ at $\rho>h$. By Theorem 2 , the horizon should be simple, so that $A \sim \rho-h$ as $\rho \rightarrow h$. Consider the function

$$
\mathcal{F}_{1}(\rho)=\frac{r^{d_{0}}}{r^{\prime}}\left[2 V-A \vec{\varphi}^{\prime 2}\right] .
$$

\footnotetext{
${ }^{3}$ The mass $M$ in conventional units, say, grams, is obtained by writing $m=G M$ where $G$ is a $\left(d_{0}+2\right)$-dimensional analogue of Newton's constant. The coefficient of $m$ is chosen in 25 and accordingly in (23) in such a way that at large $r$ in case $\Lambda=0$, when the space-time is asymptotically flat, a test particle at rest experiences a Newtonian acceleration equal to $-G M / r^{d_{0}}$.
} 
One can verify that

$$
\mathcal{F}_{1}^{\prime}(\rho)=\mathcal{F}_{2}(\rho) \stackrel{\text { def }}{=} r^{d_{0}-1}\left[2 d_{0} V+\left(d_{0}-1\right) \frac{\vec{\varphi}^{\prime 2}}{r^{\prime 2}}+A \vec{\varphi}^{\prime 2}\right] .
$$

To do so, when calculating $\mathcal{F}_{1}^{\prime}$, one should substitute $\vec{\varphi}^{\prime \prime}$ from (16), $r^{\prime \prime}$ from (18) and $A^{\prime}$ from (20). Let us integrate (27) from $h$ to infinity:

$$
\mathcal{F}_{1}(\infty)-\mathcal{F}_{1}(h)=\int_{h}^{\infty} \mathcal{F}_{2}(\rho) d \rho
$$

Since $r^{\prime}(\infty)=1$ and $r^{\prime \prime} \leq 0$, we have $r^{\prime}>1$ in the whole range of $\rho$, but $r^{\prime}(h)<\infty$. Indeed, regularity of the horizon implies a finite value of the Kretschmann scalar given by (A7), hence finite values of all its constituents (A8). In the present case, the indices $i$ and $k$ (the numbers of factor spaces) take the values 0 and 1 , and of interest for us is the quantity $R_{(3) 01}=-\frac{1}{2} A^{\prime} r^{\prime}$. Since $A^{\prime}(h)>0$, its finiteness means $r^{\prime}(h)<\infty$.

The quantity $\mathcal{F}_{1}(h)$ should be finite, since otherwise we would have either $V$ or $A \vec{\varphi}^{\prime 2}$ infinite, leading to infinite SET components (see (13)) and, via the Einstein equations, to a curvature singularity.

If, however, we admit a nonzero value of $A \vec{\varphi}^{\prime 2}$ at $\rho=h$, the integral in (28) will diverge at $\rho=h$ due to the second term in brackets in (27), and this in turn leads to an infinite value of $\mathcal{F}_{1}(h)$. Therefore $A \vec{\varphi}^{\prime 2} \rightarrow 0$ as $\rho \rightarrow h$, and we conclude that

$$
\mathcal{F}_{1}(h)=\frac{2 r^{d_{0}}(h)}{r^{\prime}(h)} V(h) \geq 0 .
$$

On the other hand, $\mathcal{F}_{1}(\infty)=0$ due to the asymptotic flatness conditions. Thus, in Eq. (28) there is a nonpositive quantity in the left-hand side and a nonnegative quantity on the right. The only way to satisfy (28) is to put $V \equiv 0$ and $\vec{\varphi}^{\prime} \equiv 0$ in the whole range $\rho>h$, and the only solution for the metric then has the Tangherlini form.

As follows from the scalar field equations $(\sqrt{16})$, the equality $V=0$ should take place where $\partial V / \partial \varphi^{K}=0$, i.e. at an extremum or saddle point of the potential, and it should be obviously a minimum for a stable equilibrium.

It is of interest that one of the key points of the above proof, that $A \vec{\varphi}^{\prime 2}=0$ at $\rho=h$, might be obtained from smoothness considerations. Indeed, since $A \sim \rho-h$ near $\rho=h$, a nonzero value of $A \vec{\varphi}^{2}$ means that some of $\left(\phi^{K}\right)^{\prime}$ behave as $(\rho-h)^{-1 / 2}$, violating the $C^{1}$ requirement for the scalar fields. Our proof is "more economical" since it only uses the requirement of space-time regularity at the horizon.

One can also note that our no-hair theorem is in a complementarity relation with a recent black hole uniqueness theorem [21] (see [22] for a review). In $D$ dimensional general relativity coupled to the $\sigma$-model (3) with $V \equiv 0$, it has been proved without assuming spherical symmetry at the outset that "the only black hole solution with a regular, non-rotating event horizon in an asymptotically flat, strictly stationary domain of outer communication is the Schwarzschild-Tangherlini solution with a constant mapping $\phi " 21$. In contrast to that, our Theorem 3 applies to $\sigma$-models with arbitrary $V(\vec{\varphi}) \geq 0$ but selects the Tangherlini solution among spherically symmetric configurations.

\subsection{Two expressions for the mass and the properties of particlelike solutions}

In this subsection we will discuss particlelike solutions, i.e., solutions with a flat asymptotic and a regular centre. We begin with a derivation of two general expressions for the active gravitational (Tangherlini) mass $m$ of a $D$-dimensional configuration with the metric (2) and an arbitrary SET compatible with the regular centre and asymptotic flatness conditions.

One expression is easily obtained from the $\left(\begin{array}{l}t \\ t\end{array}\right)$ component of Eqs. (11) which may be written in the curvature coordinates $(u=r$ in the notations of Eqs. (2), (A6)) in the following way:

$$
\frac{d_{0}}{\left(d_{0}-1\right) r^{d_{0}}} \frac{d m}{d r}=\kappa^{2} T_{t}^{t}
$$

where $m(r)$ is the mass function,

$$
m(r) \stackrel{\text { def }}{=} \frac{d_{0}-1}{2} r^{d_{0}-1}\left(1-\mathrm{e}^{-2 \alpha}\right),
$$

generalizing the well-known 4-dimensional mass function $m(r)=\frac{1}{2} r\left(1-\mathrm{e}^{-2 \alpha}\right)$. For a system with a regular centre $(r=0)$, the function $m(r)$, expressed from (29) as

$$
m(r)=\frac{d_{0}-1}{d_{0}} \kappa^{2} \int_{0}^{r} T_{t}^{t} r^{d_{0}} d r,
$$

can be interpreted as the mass inside a sphere of radius $r$. If, in addition, the space-time is asymptotically flat, this integral converges at large $r$ and, being taken from zero to infinity, gives the full Tangherlini mass $m=$ $m(\infty)$. The constant $\kappa^{2}$ is expressed in terms of $d_{0}$ and the multidimensional Newtonian constant $G$ (such that $m=G M$, see footnote (3) if we require the validity of the usual expression for mass in terms of density, $M=\int T_{t}^{t} d v$ ( $d v$ being the element of volume) in the flat space limit. One thus obtains

$$
\begin{aligned}
\kappa^{2} & =\frac{d_{0}}{d_{0}-1} s\left(d_{0}\right) G, \\
s\left(d_{0}\right) & =2 \pi^{\left(d_{0}+1\right) / 2} / \Gamma\left(\frac{d_{0}+1}{2}\right),
\end{aligned}
$$

where $s\left(d_{0}\right)$ is the area of a $d_{0}$-dimensional sphere of unit radius and $\Gamma$ is Euler's gamma function. In case $D=4$ we have, as usual, $\kappa^{2}=8 \pi G$.

Eq. (31) for the Tangherlini mass is easily rewritten in terms of any radial coordinate, e.g., the quasiglobal $\rho$ coordinate used in Eqs. (16) $-(20)$ :

$$
m=\frac{d_{0}-1}{d_{0}} \kappa^{2} \int_{\rho_{c}}^{\infty} T_{t}^{t}(\rho) r^{d_{0}} r^{\prime} d \rho,
$$


where $\rho_{c}$ is the value of $\rho$ at the centre.

On the other hand, one can integrate the $\left(\begin{array}{l}t \\ t\end{array}\right)$ component of Eqs. (12), which, in terms of the same $\rho$ coordinate (see (A6) for $R_{t}^{t}$ ), assumes the form

$$
\frac{1}{2 r^{d_{0}}}\left(A^{\prime} r^{d_{0}}\right)^{\prime}=\kappa^{2} \widetilde{T}_{t}^{t} \text {. }
$$

For an asymptotically flat metric (15) with a regular center, integration of (34) over the whole range of $\rho$ gives

$$
m=\frac{\kappa^{2}}{d_{0}} \int_{\rho_{c}}^{\infty}\left[\left(d_{0}-1\right) T_{t}^{t}-T_{i}^{i}\right] r^{d_{0}} d \rho,
$$

where the index $i$ enumerates spatial coordinates. This is a multidimensional analogue of Tolman's well-known formula [23] for the mass of a regular matter distribution in general relativity. Comparing the expressions (33) and (35), we obtain the following universal identity valid for any particlelike static, spherically symmetric configuration in D-dimensional GR:

$$
\int_{\rho_{c}}^{\infty}\left[\left(r^{\prime}-1\right)\left(d_{0}-1\right) T_{t}^{t}+T_{i}^{i}\right] r^{d_{0}} d \rho=0 .
$$

For the $\sigma$-model (3), Eq. (35) takes the form

$$
m=-\frac{2 \kappa^{2}}{d_{0}} \int_{\rho_{c}}^{\infty} V(\vec{\varphi}) r^{d_{0}} d \rho,
$$

leading to a multidimensional version of what has been previously called the generalized Rosen theorem [8]: a static, spherically symmetric particlelike solution with positive mass cannot be obtained with scalar fields having a nonnegative potential $V$.

An even stronger no-go theorem follows from the universal identity (36):

Theorem 4. Eqs. (16)-(20) with $D \geq 4$ for the $\sigma$ model (3) do not admit any particlelike solution if the matrix $H_{K L}$ is positive-definite and $V \geq 0$.

In other words, even negative-mass particlelike solutions can only be obtained with (at least partly) negative potentials.

To prove the theorem, it is sufficient to show that the expression in brackets in (36) is positive for any nontrivial solution under the conditions of the theorem. This expression is

$$
\frac{1}{2}\left(d_{0}-1\right) A\left(\vec{\varphi}^{\prime}\right)^{2}+V\left[2+\left(d_{0}-1\right) r^{\prime}\right] .
$$

Its positivity is evident since, as already mentioned, $r^{\prime}=$ 1 at the flat asymptotic and, due to $r^{\prime \prime} \leq 0$, we have $r^{\prime} \geq 1$ in the whole range of $\rho$.

\section{Theories with multiple factor spaces}

\subsection{Reduction}

Consider a $D$-dimensional static, spherically symmetric space-time $\mathbb{M}^{D}$ with the structure (6) and the metric

$$
d s_{D}^{2}=-\mathrm{e}^{2 \alpha_{0}} d u^{2}-\mathrm{e}^{2 \beta^{0}} d \Omega_{d_{0}}^{2}+\mathrm{e}^{2 \beta^{1}} d t^{2}-\sum_{i=2}^{n} \mathrm{e}^{2 \beta^{i}} d s_{i}^{2},
$$

where $d s_{i}^{2}(i=2, \ldots, n)$ are the metrics of Einstein spaces of arbitrary dimensions $d_{i}$ and signatures while $\alpha_{0}$ and all $\beta^{i}$ are functions of the radial coordinate $u$.

Consider in $\mathbb{M}^{D}$ a field theory with the action

$$
S=\int d^{D} x \sqrt{\left|g_{D}\right|}\left[\mathcal{R}_{D}+L_{\mathrm{sc}}\right]
$$

where the scalar field Lagrangian has a $\sigma$-model form similar to (3),

$$
L_{\mathrm{sc}}=h_{a b}(\bar{\phi})\left(\partial \phi^{a}, \partial \phi^{b}\right)-2 V_{D}(\bar{\phi}),
$$

$\mathcal{R}_{D}$ being the $D$-dimensional scalar curvature. The metric $h_{a b}$ of the $N^{\prime}$-dimensional target space $\mathbb{T}_{\phi}$ and the potential $V$ are functions of $\bar{\phi}=\left\{\phi^{a}\right\} \in \mathbb{T}_{\phi}$ (we use overbar s for vectors in $\mathbb{T}_{\phi}$ to distinguish them from vectors in $\mathbb{T}_{\varphi}$ labelled by arrows). The fields $\phi^{a}$ themselves are assumed to depend on the external space coordinates $x^{\mu}\left(\mu=0,1, \ldots, d_{0}+1\right)$; the notation (何) is again used, where the metric $g_{\mu \nu}$ is formed by the first three terms in (38).

The action (39) represents in a general form the scalar-vacuum sector of diverse supergravities and lowenergy limits of string and $p$-brane theories [1]. In many papers devoted to exact solutions of such lowenergy theories (see, e.g., 24] and references therein), all internal factor spaces are assumed to be Ricci-flat, and nonzero potentials $V_{D}(\bar{\phi})$ are not introduced due to technical difficulties of solving the equations. Meanwhile, the inclusion of a potential not only generalizes the theory making it possible to treat massive and/or nonlinear and interacting scalar fields, but is also necessary for describing, e.g., the symmetry breaking and Casimir effects

Let us perform a dimensional reduction to the external space-time $\mathbb{M}_{\text {ext }}$ with the metric $g_{\mu \nu}$ using the relation (A3). The action (39) is converted to

$$
\begin{aligned}
S=\int & d^{d_{0}+2} x \sqrt{\left|g_{d_{0}+2}\right|} \mathrm{e}^{\sigma_{2}}\left\{\mathcal{R}_{d_{0}+2}\right. \\
& +\sum_{i=2}^{n} d_{i}\left(d_{i}-1\right) K_{i} \mathrm{e}^{-2 \beta^{i}}+2 \nabla^{\mu} \nabla_{\mu} \sigma_{2} \\
& \left.+\sum_{i, k=2}^{n}\left(d_{i} d_{k}+d_{i} \delta_{i k}\right)\left(\partial \beta^{i}, \partial \beta^{k}\right)+L_{\mathrm{sc}}\right\},
\end{aligned}
$$

where all quantities, including the scalar $\mathcal{R}_{d_{0}+2}$, are calculated with the aid of $g_{\mu \nu}$, and

$$
\sigma_{2} \stackrel{\text { def }}{=} \sum_{d=2}^{n} d_{i} \beta^{i}
$$

so that $\mathrm{e}^{\sigma_{2}}$ is the volume factor of extra dimensions.

\footnotetext{
${ }^{4}$ On the use of effective potentials for describing the Casimir effect in compact extra dimensions, see, e.g., 25] and references therein.
} 
It is helpful to pass in the action (41), just as in the STT (5), from the Jordan-frame metric $g_{\mu \nu}$ in $\mathbb{M}_{\text {ext }}$ to the Einstein-frame metric

$$
\bar{g}_{\mu \nu}=\mathrm{e}^{2 \sigma_{2} / d_{0}} g_{\mu \nu} \text {. }
$$

After this substitution, omitting a total divergence, one obtains the action (39) in terms of $\bar{g}_{\mu \nu}$ :

$$
S=\int d^{d_{0}+2} x \sqrt{|\bar{g}|}\left[\overline{\mathcal{R}}+H_{K L}\left(\partial \varphi^{K}, \partial \varphi^{L}\right)-2 V(\vec{\varphi})\right] .
$$

Here the set of fields $\left\{\varphi^{K}\right\}=\left\{\beta^{i}, \phi^{a}\right\}$, combining the scalar fields from (40) and the moduli fields $\beta^{i}$, is treated as a vector in the extended $N=\left(n-1+N^{\prime}\right)$ dimensional target space $\mathbb{T}_{\varphi}$ with the metric

$$
\left(H_{K L}\right)=\left(\begin{array}{cc}
d_{i} d_{k} / d_{0}+d_{i} \delta_{i k} & 0 \\
0 & h_{a b}
\end{array}\right),
$$

while the potential $V(\varphi)$ is expressed in terms of $V_{D}(\bar{\phi})$ and $\beta^{i}$ :

$V(\vec{\varphi})=\mathrm{e}^{-2 \sigma_{2} / d_{0}}\left[V_{D}(\bar{\phi})-\frac{1}{2} \sum_{i=2}^{n} K_{i} d_{i}\left(d_{i}-1\right) \mathrm{e}^{-2 \beta^{i}}\right]$

We thus obtain a formulation of the theory coinciding (up to the constant $\kappa$ and the particular expression for the potential) with that discussed in Sec. 2. Therefore all results obtained in Sec. 2 are valid for the metric $\bar{g}_{\mu \nu}$ if it assumes the form (2) and the quantities $\phi^{a}$ and $\beta^{i}$ are functions of the radial coordinate $u$.

\subsection{Extended no-go theorems}

One can note that the Einstein-frame metric $\bar{g}_{\mu \nu}$ in $\mathbb{M}_{\text {ext }}$ plays an auxiliary role in our multidimensional theory with the action (39). Since the theory is not conformally invariant, the physical picture depends on the choice of a conformal frame to be regarded as a physical one. This in turn depends on the underlying fundamental theory that leads to (39) in its low-energy limit (see [26] for a discussion of physical conformal frames and further references). We do not specify such a theory, which is possibly yet unknown, therefore it seems reasonable to make the simplest choice and to consider the properties of the $D$-dimensional metric $g_{M N}$ given by (38) as a representative, conditionally physical metric. Its "external" part $g_{\mu \nu}$ is connected with $\bar{g}_{\mu \nu}$ by the conformal transformation (43). Since the action (39) corresponds to Einstein gravity in $D$ dimensions, this frame may be called the $D$-dimensional Einstein frame, and we will now call $\mathbb{M}_{\mathrm{E}}^{D}$ the manifold $\mathbb{M}^{D}$ endowed with the metric $g_{M N}$.

The quantities $\alpha_{0}, \beta^{0}, \beta^{1}$ characterizing $g_{\mu \nu}$ in (38) are connected with $\alpha, \beta, \gamma$ corresponding to $\bar{g}_{\mu \nu}$ in the form (2) as follows:

$$
\alpha_{0}=\alpha-\sigma_{2} / d_{0}, \quad \beta_{0}=\beta-\sigma_{2} / d_{0}, \quad \beta_{1}=\gamma-\sigma_{2} / d_{0},
$$

The nonminimal coupling coefficient in the action (41), being connected with the extra-dimension volume factor $\mathrm{e}^{\sigma_{2}}$, is nonnegative by definition, moreover, the solution terminates where $\mathrm{e}^{\sigma_{2}}$ vanishes or blows up. Thus, in contrast to the situation in scalar-tensor theories (see the Introduction), conformal continuations are here impossible: one cannot cross a surface, if any, where $\mathrm{e}^{\sigma_{2}}$ vanishes. Roughly speaking, due to the absence of conformal continuations, the Jordan-frame manifold $\mathbb{M}_{\text {ext }}[g]$ can be smaller but cannot be larger than $\mathbb{M}_{\text {ext }}[\bar{g}]$. More precisely, the transformation (43) establishes a one-to-one correspondence between the two manifolds if $\mathrm{e}^{\sigma_{2}}$ is regular in the whole range $\mathbb{R}_{u}$ of the radial coordinate in (2). If $\sigma_{2} \rightarrow \pm \infty$ at an intermediate value of the radial coordinate, then the transformation (43) maps $\mathbb{M}_{\text {ext }}[g]$ to only a part of $\mathbb{M}_{\text {ext }}[\bar{g}]$.

As is easily seen from the regularity conditions (A9) and (A10) presented in the Appendix, the asymptotic flatness of the metric $g_{M N}$ in $\mathbb{M}_{\mathrm{E}}^{D}$ implies an asymptotically flat Einstein-frame metric $\bar{g}_{\mu \nu}$ in $\mathbb{M}_{\text {ext }}$ and finite limits of the moduli fields $\beta^{i}, i \geq 2$, at large $r$. A similar picture is observed with the regular centre conditions: a regular centre in $\mathbb{M}_{\mathrm{E}}^{D}$ is only possible if there is a regular centre in $\mathbb{M}_{\text {ext }}[\bar{g}]$ and $\beta^{i}, i \geq 2$ behave as is prescribed in (A9). A horizon in $\mathbb{M}_{\mathrm{E}}^{D}$ always corresponds to a horizon in $\mathbb{M}_{\text {ext }}[\bar{g}]$. (The opposite assertions are not necessarily true, e.g., a regular centre in $\mathbb{M}_{\text {ext }}[\bar{g}]$ may be "spoiled" when passing to $g_{M N}$ by an improper behaviour of the moduli fields $\beta^{i}$.)

So the global properties of $\mathbb{M}_{\text {ext }}[\bar{g}]$ and $\mathbb{M}_{\text {ext }}[g]$ (and hence $\mathbb{M}_{\mathrm{E}}^{D}$ ) are closely related but not entirely coincide.

Let us describe some essential features of $\mathbb{M}_{\text {ext }}[g]$ and $\mathbb{M}_{\mathrm{E}}^{D}$, associated with Statements A-D in the Introduction.

A. The no-hair theorem can be formulated for $\mathbb{M}_{\mathrm{E}}^{D}$ as follows:

Theorem 5. Given the action (39), (40), with $h_{a b}$ positive-definite and a nonnegative potential (46), in the space-time $\mathbb{M}_{\mathrm{E}}^{D}$ with the metric (38), the only static, asymptotically flat black hole solution to the field equations is characterized in the region of outer communication by $\phi^{a}=$ const, $\beta^{i}=$ const $(i=\overline{2, n}), V(\vec{\varphi}) \equiv 0$ and the Tangherlini metric $g_{\mu \nu}$.

In other words, the only asymptotically flat black hole solution is given by the Tangherlini metric in $\mathbb{M}_{\text {ext }}$, constant scalar fields $\phi^{a}$ and constant moduli fields $\beta^{i}$ outside the event horizon. Note that in this solution the metrics $g_{\mu \nu}$ and $\bar{g}_{\mu \nu}$ in $\mathbb{M}_{\text {ext }}$ are connected by simple scaling with a constant conformal factor since $\sigma_{2}=$ const .

Another feature of interest is that it is the potential (46) that vanishes in the black hole solution rather than the original potential $V_{D}(\bar{\phi})$ from Eq. (40). If all internal factor spaces are Ricci-flat, then both $V_{D}(\bar{\phi})$ and $V(\vec{\varphi})$ are zero in a black hole solution. If not, then the curvatures of the internal factor spaces compensate 
one another or/and the potential $V_{D}(\bar{\phi})$. The latter, if nonzero, is in this case necessarily constant, appearing as a cosmological constant in the action (39).

Theorem 5 generalizes Theorem 3 from Sec. 2 and also the previously known property of black holes with the metric (38) when the internal spaces are Ricci-flat and the source is a massless, minimally coupled scalar field without a potential [27].

B. Particlelike solutions: Theorem 4 is valid in $\mathbb{M}_{\mathrm{E}}^{D}$ in the same formulation, but the condition $V \geq 0$ mentioned there applies to the potential (46) rather than $V_{D}(\bar{\phi})$ from $(40)$.

One can note that the narrower formulation of the generalized Rosen theorem involving the sign of mass could not be so easily extended to the metric $g_{\mu \nu}$ since the mass value is, in general, sensitive to conformal transformations.

C. Wormholes and even wormhole throats are impossible with the metric $\bar{g}_{\mu \nu}$. The conformal factor $\mathrm{e}^{2 \sigma_{2} / d_{0}}$ in (43) removes the prohibition of throats since for $g_{\mu \nu}$ a condition like $r^{\prime \prime} \leq 0$ (see Eq. (18)) is no longer valid. However, a wormhole as a global entity with two flat asymptotics cannot appear in $\mathbb{M}_{J}=\mathbb{M}_{\text {ext }}\left[g_{\mu \nu}\right]$. Indeed, if we suppose the contrary, then, due to the correspondence between flat asymptotics of the two metrics, we immediately obtain a wormhole in $\mathbb{M}_{\mathrm{E}}=\mathbb{M}_{\text {ext }}\left[\bar{g}_{\mu \nu}\right]$, forbidden by Theorem 1 .

Flux-tube solutions with nontrivial scalar and/or moduli fields are absent, as before, but horns are not ruled out since the behaviour of the metric coefficient $g_{\theta \theta}$ is modified by conformal transformations.

Let us emphasize that all the restrictions mentioned in items A-C are invalid if the target space metric $h_{a b}$ is not positive-definite.

D. The global causal structure of any Jordan frame cannot be more complex than that of the Einstein frame even in STT, where conformal continuations are allowed [10]. The reasoning of [10 entirely applies to $\mathbb{M}_{\text {ext }}[\mathrm{g}]$ and hence to $\mathbb{M}_{\mathrm{E}}^{D}$. The list of possible global structures is again the same as that for the Tangherlini-de Sitter metric (23). This restriction does not depend (i) on the choice and even sign of scalar field potentials, (ii) on the nature of asymptotic conditions and (iii) on the algebraic properties of the target space metric. Let us recall that in STT it was also proved to be conformal frame independent, regardless of possible conformal continuations. It is therefore the most universal property of spherically symmetric configurations with scalar fields in various theories of gravity.

A theory in $\mathbb{M}^{D}$ may, however, be initially formulated in another conformal frame, i.e., with a nonminimal coupling factor $f(\bar{\phi})$ before $\mathcal{R}_{D}$ in (39). Let us designate $M^{D}$ in this case as $\mathbb{M}_{\mathrm{J}}^{D}$, a $D$-dimensional Jordan-frame manifold. (An example of such a construction is the so-called string metric in string theories [1] where $f$ depends on a dilaton field related to string coupling.) Applying a conformal transformation like (7), we can recover the Einstein-frame action (39) in $\mathbb{M}_{\mathrm{E}}^{D}$, then by dimensional reduction pass to $\mathbb{M}_{\text {ext }}[g]$ and after one more conformal mapping (43) arrive at the $\left(d_{0}+2\right)$ Einstein frame $\mathbb{M}_{\text {ext }}[\bar{g}]$. Addition of the first step in this sequence of reductions weakens our conclusions to a certain extent. The main point is that we cannot a priori require $f(\bar{\phi})>0$ in the whole range of $\vec{\varphi}$, therefore conformal continuations (CCs) through surfaces where $f=0$ are not excluded.

Meanwhile, the properties of CCs have been studied in [10] only for a single scalar field in $\mathbb{M}_{\text {ext }}$ (in the present notation). In our more complex case of multiple scalar fields and factor spaces, such a continuation through the surface $f(\bar{\phi})=0$ in the multidimensional target space $\mathbb{T}_{\phi}$ can have yet unknown properties.

One can only say for sure that the no-hair and nowormhole theorems fail if CCs are admitted. This follows from the simplest example of CCs in the solutions with a conformal scalar field in GR, leading to black holes [28, 29] and wormholes [12, 30] and known since the 70s although the term "conformal continuation" was introduced only recently 9]. Let us also recall that a wormhole was shown to be one of the generic structures appearing as a result of CCs in scalar-tensor theories [10].

If we require that the function $f(\bar{\phi})$ should be finite and nonzero in the whole range $\mathbb{R}_{u}$ of the radial coordinate, including its ends, then all the above no-go theorems are equally valid in $\mathbb{M}_{\mathrm{E}}^{D}$ and $\mathbb{M}_{\mathrm{J}}^{D}$. One should only bear in mind that the transformation (7) from $\mathbb{M}_{\mathrm{E}}^{D}$ to $\mathbb{M}_{J}^{D}$ modifies the potential $V_{D}(\bar{\phi})$ multiplying it by $f^{-D /(D-2)}$, which in turn affects the explicit form of the condition $V \geq 0$, essential for Statements A and B.

Statement D on possible horizon dispositions and global causal structures will be unaffected if we admit an infinite growth or vanishing of $f(\bar{\phi})$ at the extremes of the range $\mathbb{R}_{u}$. However, Statement $\mathrm{C}$ will not survive: such a behaviour of $f$ may create a wormhole or horn in $\mathbb{M}_{\mathrm{J}}^{D}$. A simple example of this kind is a "horned particle" in the string metric in dilaton gravity of string origin, studied by Banks et al. [14.

\section{Appendix.}

\section{Some geometric quantities for $D$-dimensional space-times}

Consider a $D$-dimensional space-time $\mathbb{M}^{D}$ with the metric

$$
d s_{D}^{2}=g_{M N} d x^{M} d x^{N}=g_{\mu \nu} d x^{\mu} d x^{\nu}-\sum_{i=2}^{n} \mathrm{e}^{2 \beta^{i}} d s_{i}^{2}
$$

where the indices $M, N, \ldots=\overline{0, D-1}$, the indices $\mu, \nu$ refer to the external space $\mathbb{M}_{\text {ext }}$, while $g_{\mu \nu}$ and $\beta^{i}$ are functions of $x^{\mu}$. The internal factor spaces $\mathbb{M}_{i}(i=$ $2, \ldots, n)$ of arbitrary dimensions $d_{i}$ and signatures, with $x^{\mu}$-independent metrics $d s_{i}^{2}$, are assumed to be Einstein spaces, so that the corresponding Ricci tensors can be 
written as

$$
\mathcal{R}_{m_{i}}^{n_{i}}=K_{i}\left(d_{i}-1\right) \delta_{m_{i}}^{n_{i}}
$$

where $K_{i}=+1,0,-1$ for spaces of positive, zero and negative curvature, respectively. The actual values of their curvatures depend on the corresponding scale factors $\mathrm{e}^{2 \beta^{i}}$ in (A1).

The scalar curvature of $\mathbb{M}^{D}$ with the metric (A1) can be expressed in terms of the scalar curvature $\mathcal{R}_{d_{0}+2}$ of the external subspace $\mathbb{M}_{\text {ext }}$ and the functions $\beta^{i}$ :

$$
\begin{gathered}
\mathcal{R}_{D}=\mathcal{R}_{d_{0}+2}+\sum_{i=2}^{n} d_{i}\left(d_{i}-1\right) \mathrm{e}^{-2 \beta^{i}} \\
+2 \sum_{i=2}^{n} d_{i} \nabla^{\mu} \nabla_{\mu} \beta^{i}+\sum_{i, k=2}^{n}\left(d_{i} d_{k}+d_{i} \delta_{i k}\right)\left(\partial \beta^{i}, \partial \beta^{k}\right)
\end{gathered}
$$

where all quantities, including the Ricci scalar $\mathcal{R}_{d_{0}+2}$, are calculated with the aid of $g_{\mu \nu}$.

If the external space-time is static, spherically symmetric with the structure $\mathbb{M}_{\text {ext }}=\mathbb{R}_{u} \times \mathbb{M}_{0} \times \mathbb{M}_{1}$ and the metric (2) (with the identifications $\alpha=\alpha_{0}, \gamma=\beta^{1}$ and $\beta=\beta^{0}$ ), where $\mathbb{R}_{u}$ is the range of the radial coordinate $u$, then the coordinate spheres $M_{0}=\mathbb{S}^{d_{0}}$ and the time axis $\mathbb{M}_{1}$ are included in the general scheme, so that $K_{0}=+1, K_{1}=0$ and $d_{1}=1$, and the metric can be written as

$$
d s_{D}^{2}=-\mathrm{e}^{2 \alpha_{0}} d u^{2}+\sum_{i=0}^{n} \mathrm{e}^{2 \beta^{i}} d s_{i}^{2} .
$$

In case $n=1$, internal factor spaces are absent, and we return to the structure $\mathbb{M}^{D}=\mathbb{M}_{\text {ext }}=\mathbb{R}_{u} \times \mathbb{M}_{0} \times \mathbb{M}_{1}$.

Nonzero Ricci tensor components for the spherically symmetric metric A4 are

$$
\begin{aligned}
\mathcal{R}_{u}^{u}= & -\sum_{i=0}^{n} \mathrm{e}^{-2 \alpha_{0}}\left(\ddot{\beta}^{i}+\dot{\beta}^{i 2}-\dot{\alpha}_{0} \dot{\beta}^{i}\right), \\
\mathcal{R}_{n_{i}}^{m_{i}}= & \delta_{n_{i}}^{m_{i}}\left\{\left(d_{i}-1\right) K_{i} \mathrm{e}^{-2 \beta^{i}}\right. \\
& \left.-\mathrm{e}^{-2 \alpha_{0}}\left[\ddot{\beta}^{i}+\dot{\beta}^{i}\left(-\dot{\alpha}_{0}+\sum_{k=0}^{n} d_{k} \dot{\beta}^{k}\right)\right]\right\},
\end{aligned}
$$

where the dots denote $d / d u$ and the indices $m_{i}, n_{i}$ belong to coordinates from the $i$-th factor space.

From these general expressions, putting $n=1$, it is easy to obtain the Ricci tensor components in the particular gauge $\alpha_{0}+\beta^{1}=0$ (the quasiglobal coordinate $\rho$ ) for the metric (15) used in Sec. 2:

$$
\begin{aligned}
& \mathcal{R}_{t}^{t}=-\frac{1}{2 r^{d_{0}}}\left(A^{\prime} r^{d_{0}}\right)^{\prime} ; \\
& \mathcal{R}_{\rho}^{\rho}=-\frac{1}{2 r^{d_{0}}}\left(A^{\prime} r^{d_{0}}\right)^{\prime}-d_{0} A \frac{r^{\prime \prime}}{r} ; \\
& \mathcal{R}_{\theta}^{\theta}=\frac{d_{0}-1}{r^{2}}-A\left[\frac{r^{\prime \prime}}{r}+\left(d_{0}-1\right) \frac{r^{\prime 2}}{r^{2}}+\frac{r^{\prime}}{r} \frac{A^{\prime}}{A}\right],
\end{aligned}
$$

where $A=\mathrm{e}^{2 \beta^{1}}, r=\mathrm{e}^{\beta^{0}}, \theta$ is any of the angular coordinates parametrizing the sphere $\mathbb{S}^{d_{0}}$ and the prime denotes $d / d \rho$.
Let us now return to the metric (A4) and give an expression for its Kretschmann scalar (Riemann tensor squared) $\mathcal{K}=\mathcal{R}_{M N P Q} \mathcal{R}^{M N P Q}$ :

$\mathcal{K}=4 \sum_{i} R_{(1) i}^{2}+2 \sum_{i} d_{i}\left(d_{i}-1\right) R_{(2) i}^{2}+4 \sum_{i \neq k} d_{i} d_{k} R_{(3) i k}^{2}$

where $i, k=\overline{0, n}$ and

$$
\begin{aligned}
R_{(1) i} & =-\mathrm{e}^{-2 \alpha}\left(\ddot{\beta}^{i}-\dot{\alpha} \dot{\beta}^{i}+\dot{\beta}^{i 2}\right), \\
R_{(2) i} & =K_{i} \mathrm{e}^{-2 \beta^{i}}-\mathrm{e}^{-2 \alpha} \dot{\beta}^{i 2}, \\
R_{(3) i k} & =-\mathrm{e}^{-2 \alpha} \dot{\beta}^{i} \dot{\beta}^{k} ;
\end{aligned}
$$

as before, the dots denote $d / d u$ (recall that $u$ is an arbitrary radial coordinate, not to be confused with the particular coordinate $\rho$ in Eqs. (A6)).

The expression (A7) is a sum of squares of the Riemann tensor components $\mathcal{R}_{\mu \nu}{ }^{\lambda \sigma}$, hence its proper behaviour at the centre or any other point guarantees the regularity properties of the manifold $\mathbb{M}^{D}$. Thus, a centre is by definition a place where the coordinate spheres are drawn to points, i.e., $r=\mathrm{e}^{\beta_{0}} \rightarrow 0$. The regular centre conditions follow from the requirement $\mathcal{K}<\infty$ at such a value of the coordinate $u$ :

$$
\beta^{i}=\beta_{0}^{i}+O\left(r^{2}\right), \quad i=\overline{1, n} ; \quad \mathrm{e}^{-2 \alpha} \dot{r}^{2}=1+O\left(r^{2}\right),
$$

where $\beta_{0}^{i}$ are constants. The last condition is the local euclidity requirement, providing a correct circumference to radius ratio for circles around the centre; it follows from the requirement of finiteness of $R_{(2) 0}$ at $r=0$.

The metric (A4) can be called asymptotically flat when its external part is asymptotically flat and the internal scale factors $\mathrm{e}^{\beta^{i}}$ tend to finite constant values as $r \rightarrow \infty$. Since $\beta^{i}$ behave as effective scalar fields in $\mathbb{M}_{\text {ext }}$, the asymptotic flatness of $\mathbb{M}_{\text {ext }}$ requires a sufficiently rapid decay of $\dot{\beta}^{i}$ at infinity. We thus require

$$
\beta^{i}=\beta_{\infty}^{i}+O(1 / r), \quad i=\overline{1, n} ; \quad \mathrm{e}^{-2 \alpha} \dot{r}^{2} \rightarrow 1 .
$$

where $\beta_{\infty}^{i}$ are constants. The last condition in A10 is the requirement of a correct circumference to radius ratio for circles $r=$ const as $r \rightarrow \infty$; it rules out the possibility of asymptotics of conical nature. Under these conditions, all components of the Riemann tensor manifestly vanish at infinity.

The conditions (A9) and (A10) are written for an arbitrary radial coordinate $u$ but may be easily reformulated for its particular choice. Thus, for the quasiglobal coordinate $\rho$ one can assume $r \approx \rho$ at large $r$, then the last condition in (A10) reads simply $\mathrm{e}^{-2 \alpha_{0}} \equiv A \rightarrow 1$ as $\rho \rightarrow \infty$. The central value of $\rho$ is $\rho_{c}$, not necessarily zero; as follows from the first condition (A9), $A\left(\rho_{c}\right)=A_{c}$ is finite, while the last condition yields $A(d r / d \rho)^{2}=1+O\left(\left(\rho-\rho_{c}\right)^{2}\right)$ near the centre. 


\section{Acknowledgements}

We are grateful to Irina Dymnikova and Vitaly Melnikov for helpful discussions. KB and SF acknowledge partial financial support from the Russian Foundation for Basic Research and the Russian Ministry of Industry, Science and Technologies.

\section{References}

[1] A. Salam and E. Sezgin, eds., "Supergravities in Diverse Dimensions", reprints in 2 vols., World Scientific, 1989; M.B. Green, J.H. Schwarz, and E. Witten, "Superstring Theory" in 2 vols., Cambridge Univ. Press, 1987; K.S. Stelle, "Lectures on supergravity p-branes ", hep$\operatorname{th} / 9701088$

M.J. Duff, "M-theory (the theory formerly known as strings)", hep-th/9608117.

[2] N. Khviengia, Z. Khviengia, H. Lü and C.N. Pope, "Toward a field theory of F-theory", Class. Quantum Grav. 15, 759 (1998); hep-th/9703012;

I. Bars, Phys. Lett. 403B, 257 (1997);

A.M. Gavrilik, Acta Phys. Acad. Sci. Hung. 11, 35 (2000).

[3] J.D. Bekenstein, Phys. Rev. D 5, 1239 (1972); ibid., 2403; "Black holes: classical properties, thermodynamics, and heuristic quantization", gr-qc/9808028 (review).

[4] S. Adler and R.B. Pearson, Phys. Rev. D 18, 2798 (1978).

[5] K.A. Bronnikov and G.N. Shikin, "Self-gravitating particle models with classical fields and their stability". Series "Itogi Nauki i Tekhniki" ("Results of Science and Engineering"), Subseries "Classical Field Theory and Gravitation Theory", v. 2, p. 4, VINITI, Moscow 1991 (in Russian).

[6] K.A. Bronnikov, Phys. Rev. D 64, 064013 (2001).

[7] K.C.K. Chan, J.H. Horne and R.B. Mann, Nucl. Phys. B 447, 441 (1995).

[8] K.A. Bronnikov and G.N. Shikin, Grav. \& Cosmol 8, 107 (2002); gr-qc/0109027.

[9] K.A. Bronnikov, Acta Phys. Polon. B32, 3571 (2001); gr-qc/0110125.

[10] K.A. Bronnikov, J. Math. Phys. 43, No. 12 (2002), grqc/0204001.

[11] R. Wagoner, Phys. Rev. D 1, 3209 (1970).

[12] K.A. Bronnikov, Acta Phys. Polon. B4, 251-273 (1973).

[13] K.A. Bronnikov, G. Clément, C.P. Constantinidis and J.C. Fabris, Phys. Lett. 243A, 121 (1998), grqc/9801050; Grav. \& Cosmol. 4, 128 (1998), grqc/9804064

[14] T. Banks, A. Dabholkar, M.R. Douglas and M. O'Loughlin, Phys. Rev. D 45, 3607 (1992);

T. Banks and M. O'Loughlin, Phys. Rev. D 47, 540 (1993).

[15] M. Walker, J. Math. Phys. 11, 8, 2280 (1970).

[16] K.A. Bronnikov, Izv. Vuzov, Fizika No. 6, 32 (1979).
[17] M.O. Katanaev, Nucl. Phys. Proc. Suppl. 88, 233-236 (2000), gr-qc/9912039; Proc. Steklov Inst. Math. 228, 158-183, gr-qc/9907088.

[18] T. Klosch and T. Strobl, Class. Quantum Grav. 13, 2395-2422 (1996); 14, 1689-1723 (1997).

[19] F.R. Tangherlini, Nuovo Cim. 77, 636 (1963).

[20] K. Lake and R. Roeder, Phys. Rev. D 15, 3513 (1977); M. Katanaev, T. Klosch and W. Kummer. Ann. Phys. (USA) 276, 191 (1999).

[21] M. Rogatko, "Uniqueness theorem for static black hole solutions of $\sigma$-models in higher dimensions", hepth/0207187.

[22] M. Heusler, "Black Hole Uniqueness Theorems". Cambridge Univ. Press, 1997;

P.O. Mazur, "Black Hole Uniqueness Theorems", hepth/0101012.

[23] R.C. Tolman, "Relativity, Thermodynamics and Cosmology", Clarendon Press, Oxford, 1969.

[24] K.A. Bronnikov, V.D. Ivashchuk and V.N. Melnikov, gr-qc/9710054; Grav. and Cosmol. 3, 203 (1997);

K.A. Bronnikov, hep-th/9710207; Grav. and Cosmol. 4, 49 (1998);

V.D. Ivashchuk and V.N. Melnikov, Class. Quantum Grav. 18, R87-R152 (2001).

[25] U. Günther and A. Zhuk, Phys. Rev. D 56, 6391 (1997); U. Günther, S. Kriskiv and A. Zhuk, Grav. \& Cosmol. 4, 1 (1998).

[26] K.A. Bronnikov and V.N. Melnikov, gr-qc/0103079, Gen. Rel. Grav. 33, 1549 (2001).

[27] S.B. Fadeev, V.D. Ivashchuk and V.N. Melnikov, Phys. Lett. 161A, 98 (1991).

[28] N.M. Bocharova, K.A. Bronnikov and V.N. Melnikov, Vestn. Mosk. Univ., Fiz. Astron. No. 6, 706 (1970).

[29] J.D. Bekenstein, Ann. Phys. (USA) 82, 535 (1974).

[30] C. Barceló and M. Visser, Phys. Lett. 466B, 127-134 (1999). 